\title{
Recent Developments SAXS using MetalJet X-ray Source
}

\author{
A Adibhatla ${ }^{1}$ \\ ${ }^{1}$ Excillum Inc, Grafton, MA \\ anasuya.adibhatla@excillum.com
}

High-end x-ray scattering techniques such as SAXS, BIO-SAXS, non-ambient SAXS and GISAXS rely heavily on the $\mathrm{x}$-ray source brightness for resolution and exposure time. Traditional solid or rotating anode $\mathrm{x}$-ray tubes are typically limited in brightness by when the e-beam power density melts the anode. The liquid-metal-jet technology has overcome this limitation by using an anode that is already in the molten state. With bright compact sources, time resolved studies could be achieved even in the home laboratory. We report brightness of $6.5 \times 1010$ photons $/(\mathrm{s} \cdot \mathrm{mm} 2 \cdot \operatorname{mrad} 2 \cdot$ line $)$ over a spot size of $10 \mu \mathrm{m} \mathrm{FWHM}$. Over the last years, the liquid-metal-jet technology has developed from prototypes into fully operational and stable X-ray tubes running in more than 8 labs over the world. Multiple users and system manufacturers have been now routinely using the metal-jet anode x-ray source in high-end SAXS set-ups. With the high brightness from the liquid-metal-jet x-ray source, novel techniques that was only possible at synchrotron before can now also be used in the home lab. Examples involving in-situ measurements and time resolution such as SEC-SAXS or growth kinetics with temporal resolution on the order of seconds will be shown. This presentation will review the current status of the metal-jet technology specifically in terms of stability, lifetime, flux and optics. It will furthermore refer to some recent SEC-SAXS and bio-SAXS data from users.

Acta Cryst. (2020). A76, a155 